The change in Profile of Fatigue Related States (PFRS) score was similar in men with a significant decrease $(-45 \%)$ as in women $(-52 \%)$ (F 4.8, p < 0.001).

None of the individuals had any prior diagnosis of chronic fatigue syndrome.

All were new attendees to the clinic at the time of initial assessment.

Conclusion. Our findings indicate that this intervention based on massage and mobility exercises significantly reduced fatigue related to Long COVID.

It may be that early intervention and supportive treatments at the end of the acute phase of COVID-19 can help overcome acute phase symptoms and prevent them becoming chronic/enduring.

Does food responsiveness change in people with first episode psychosis (FEP) over a period of 6 months after commencing antipsychotics? Preliminary results

Adrian Heald $^{1 *}$, Mark Shakespeare ${ }^{2}$, Kevin Williamson ${ }^{2}$, Adrianne Close ${ }^{2}$, Adrian Phillipson ${ }^{2}$ and Suzanne Higgs ${ }^{3}$

${ }^{1}$ Salford Royal Foundation Trust; ${ }^{2}$ RDASH and ${ }^{3}$ University of Birmingham

${ }^{\star}$ Corresponding author.

doi: 10.1192/bjo.2021.682

Aims. We here present preliminary results from our study to understand better the changes in people' s experience of food in the months after diagnosis with first episode psychosis (FEP). Weight gain often occurs in the weeks/months after diagnosis and is related to an increase in appetite and food intake. Many drugs that are effective in treating psychosis are associated with changes in the way that people experience reward when they eat.

The aim of this project is to increase our understanding of exactly why this happens in terms of an individual's experience of food reward and reduced satiety - and therefore how we can help people with FEP to keep their weight down. At this stage we are looking at the feasibility of applying currently available evaluation tools to people in this situation.

Method. A convenience sample was used to recruit 10 service users from RDaSH NHS FT Early Intervention Services. This is a feasibility study which will provide data to underpin a fully powered, larger trial.

Rating scales applied were:

Power of food questionnaire: measures responsiveness to the food environment.

Intuitive Eating Scale: measures an individual's tendency to follow their physical hunger and satiety cues.

The loss of control over eating scale (LOCES): measures a global sense of whether individuals experience LOC over eating.

Dutch Eating Behaviour Questionnaire (DEBQ): measures restrained eating, emotional eating and external eating.

Result. The ages of the participants ranged from 17-26 years. All were started on Olanzapine at the dose of 5 or $10 \mathrm{mg}$ daily.

Baseline total scores for the Power of Food (2.47-3.80)/5 (higher score $=$ more responsiveness) and Intuitive Eating scales $(2.10-2.62) / 5$ (higher score $=$ greater tendency to follow hunger and satiety cues) were in the mid-range, while the LOCES scores varied widely from $1.50-2.38 / 5$.

The DEBQ restrained subscale score range was 2.40-2.80/5 (higher indicates greater restraint with food) while the DEBQ external subscale ranged from $2.70-3.00 / 5$ (higher = greater tendency to overeat) and the DEBQ emotional subtotal score was $1.92-1.94 / 5$, in keeping with a relatively low emotional drive to eat.
Conclusion. Our preliminary results reveal at the beginning of antipsychotic treatment a moderate responsiveness to food and tendency to follow hunger/ satiety cues, with scores for Loss of Control of eating in the low to moderate range and a low emotional drive to eat. The difference between these and the follow-up eating behaviour scores will provide important clues as to the precise changes in eating behaviour with anti-psychotic treatment in FEP.

\section{Reduced motivation to work for financial reward associated with harmful alcohol use in a community sample of young adults}

\section{Jessica Henry*, Karen Ersche and Tsen Vei Lim}

Department of Psychiatry, University of Cambridge ${ }^{\star}$ Corresponding author.

\section{doi: 10.1192/bjo.2021.683}

Aims. Neuroimaging research suggests that alcohol dependency is associated with impairments in anticipating monetary rewards, but not aversive or alcohol-related cues.

We sought to investigate if reinforcement sensitivity is altered in young adults, who regularly consume harmful levels of alcohol, using a monetary incentive reinforcement (MIR) task. In light of previous research suggesting reduced motivation to obtain reward, we hypothesized that young alcohol users would show reduced motivation for monetary gain, but unimpaired loss avoidance behaviour.

Method. We recruited 46 volunteers from the local community in Cambridge (UK), half of whom reported consuming alcohol at harmful levels, as reflected by the Alcohol Use Disorder Test. Participants completed a number of personality questionnaires, including the Barratt Impulsivity Scale (BIS-11) and Sensation-Seeking-Scale (SSS-V) and performed the MIR task, which measures participants' efforts in avoiding punishment and gaining rewards. Data were analysed using Statistical Package for Social Sciences (SPSS) version 25 (IBM, Chicago IL). Analysis of co-variance (ANOVA) were used to explore group differences in demographics, personality traits and task performance; age and gender were included as co-variates.

Result. The groups were well-matched in terms of socioeconomic status and education levels. As the alcohol group was significantly younger than the control group and dominated by females, age and gender were statistically controlled for. Alcohol users reported significantly higher levels of impulsivity $(\mathrm{F} 1,41=6.0$, $\mathrm{p}=0.019)$ and sensation-seeking traits $(\mathrm{F} 1,42=36.7, \mathrm{p}<0.001)$ and demonstrated normal sensitivity to monetary value (F1,41 $=1.07, \mathrm{p}=0.307)$. However, when challenged to on the MIR task to gain reward or avoid punishment, alcohol users were as equally motivated as control volunteers to take action to avoid financial loss $(\mathrm{F} 1,41=2.6, \mathrm{p}=0.112)$ but showed less motivation to work towards financial reward $(\mathrm{F} 1,41=4.7, \mathrm{p}=0.036)$. Especially for small rewards, alcohol users exerted significantly less efforts, as reflected by a reduced accuracy rate $(\mathrm{F} 1,41=6.6, \mathrm{p}=0.014)$ and a significant increase in late responses $(\mathrm{F} 1,41=7.7, \mathrm{p}=0.008)$. The lack of motivation to work for reward was negatively associated with the severity of alcohol use, as reflected by the AUDIT score $(\mathrm{r}=-.48, \mathrm{p}<0-05)$.

Conclusion. We observed reduced motivation to obtain financial reward, but not avoid loss in a community sample of heavy drinkers. As the observed effect was directly related to alcohol use severity, it may suggest changes in reinforcement sensitivity occur at an early stage of chronic alcohol use. Future research may want to monitor reward motivation in alcohol users longitudinally to evaluate whether it would be a suitable target for early intervention. 